\title{
Riscos ergonômicos no cotidiano dos profissionais de enfermagem dos hospitais
}

\section{brasileiros}

\author{
Ergonomic risks in the everyday of nursing professionals in brazilian hospitals \\ Riesgos ergonómicos en la vida cotidiana de los profesionales de enfermería de los hospitales \\ brasileños
}

Recebido: 18/05/2021 | Revisado: 25/05/2021 | Aceito: 06/06/2021 | Publicado: 2106/2021

Thales Eduardo Rodrigues Alpi ORCID: https://orcid.org/0000-0002-8248-2028

Universidade Franciscana, Brasil E-mail: thalesalpi@ufn.edu.br

Pabline Pivetta de Oliveira ORCID: https://orcid.org/0000-0001-5708-9075 Universidade Franciscana, Brasil E-mail: pablinepivetta@gmail.com

Regina Gema Santini Costenaro ORCID: https://orcid.org/0000-0001-8657-2066 Universidade Franciscana, Brasil E-mail: reginacostenaro@gmail.com Rosiane Filipin Rangel ORCID: https://orcid.org/0000-0003-4059-4176 Universidade Franciscana, Brasil E-mail: rosifrangel@gmail.com

Silomar Ilha

ORCID: https://orcid.org/0000-0002-2132-9505 Universidade Franciscana, Brasil E-mail: silo_sm@hotmail.com

\begin{abstract}
Resumo
Objetivou-se, identificar as causas e ameaças mais frequentes aos profissionais de enfermagem do setor hospitalar, no que diz respeito ao risco de saúde ocupacional. Trata-se de uma revisão narrativa da literatura, a busca ocorreu no mês de junho/2019, e foram avaliados materiais digitais no banco de dados do Observatório de segurança e saúde do trabalho, tais como: documentos do Ministério da Economia, Secretária e Inspeção do trabalho e, documento do Ministério da Saúde. Também foram selecionados, 10 estudos relacionados a ergonomia em profissionais de enfermagem. Análise dos materiais mostram dados relativos a Distribuição Geográfica das Comunicações de Acidentes de Trabalho; Estimativa de subnotificação de acidentes de trabalho; Setores econômicos com maiores números de notificações; Ocupações dentro do setor hospitalar; Despesas previdenciárias-auxílio doença por acidentes de trabalho; Lesões mais frequentes; Partes do corpo mais frequentemente atingidas; Partes do corpo mais frequentemente atingidas pelo esforço físico; e, o Afastamento do ambiente laboral conforme a classificação Internacional de doenças. Identificou-se que aproximadamente $60 \%$ dos casos de acidentes ergonômicos dentro da unidade hospitalar são sofridos por profissionais de enfermagem. Quanto as causas mais frequentes de afastamentos dos profissionais do ambiente hospitalar, destacaram-se as lesões osteomusculares e de tecido conjuntivo, seguida de fraturas, doenças mentais e comportamentais, bem como luxações. Quanto as partes do corpo mais frequentemente atingidas, destacaram-se os dedos. Assim, faz-se necessário maior ênfase na sensibilização dos gestores e dos trabalhadores sobre riscos ocupacionais e suas consequências, com vistas a reduzir a exposição a riscos e possíveis danos à saúde.
\end{abstract}

Palavras-chave: Hospitais; Ergonomia; Saúde do trabalhador; Enfermagem.

\begin{abstract}
The objective was to identify the most frequent causes and threats to nursing professionals in the hospital sector, with regard to occupational health risk. This is a narrative review of the literature, the search took place in June/2019, and digital materials were evaluated in the database of the Occupational Safety and Health Observatory, such as: documents from the Ministry of Economy, Secretary and Inspection of work and a document from the Ministry of Health. 10 studies related to ergonomics in nursing professionals were also selected. Analysis of the materials show data related to the Geographical Distribution of Work Accident Communications; Estimate of underreporting of accidents at work; Economic sectors with higher numbers of notifications; Occupations within the hospital sector; Social security expenses-sickness allowance for work accidents; Most frequent injuries; Parts of the body most
\end{abstract}


frequently affected; Parts of the body most frequently affected by physical exertion; and, Removal from the work environment according to the International Classification of Diseases. It was identified that approximately $60 \%$ of cases of ergonomic accidents within the hospital are suffered by nursing professionals. As for the most frequent causes of absence of professionals from the hospital environment, musculoskeletal and connective tissue injuries stood out, followed by fractures, mental and behavioral diseases, as well as dislocations. As for the most frequently affected parts of the body, the fingers stood out. Thus, greater emphasis is needed on sensitizing managers and workers about occupational hazards and their consequences, with a view to reducing exposure to risks and possible damage to health.

Keywords: Hospitals; Ergonomics; Occupational health; Nursing.

\section{Resumen}

El objetivo de este estudio era identificar las causas y amenazas más frecuentes a los profesionales de enfermería del sector hospitalario, en lo que respecta al riesgo para la salud en el trabajo. Esta es una revisión narrativa de la literatura, la búsqueda tuvo lugar en junio/2019, y los materiales digitales fueron evaluados en la base de datos del Observatorio de Seguridad y Salud en el Trabajo, tales como: documentos del Ministerio de Economía, Secretario e Inspección de Trabajo y, documento del Ministerio de Salud. También seleccionamos 10 estudios relacionados con la ergonomía en profesionales de enfermería. El análisis de los materiales muestra datos relacionados con la Distribución Geográfica de las Comunicaciones de Accidentes Laborales; stimación de la subregistro de accidentes de trabajo; Sectores económicos con mayor número de notificaciones; Ocupaciones dentro del sector hospitalario; Ayudas a los gastos de la Seguridad Social por enfermedad debidas a accidentes de trabajo; Lesiones más frecuentes; Partes del cuerpo más afectadas; Partes del cuerpo más a menudo afectadas por el esfuerzo físico; y la eliminación del entorno de trabajo de acuerdo con la Clasificación Internacional de Enfermedades. Se identificó que aproximadamente el $60 \%$ de los casos de accidentes ergonómicos dentro de la unidad hospitalaria son sufridos por profesionales de enfermería. En cuanto a las causas más frecuentes de expulsión de profesionales del ámbito hospitalario, destacaron las lesiones osteomusculares y del tejido conjuntivo, seguidas de las fracturas, enfermedades mentales y del comportamiento, así como luxaciones. En cuanto a las partes del cuerpo más frecuentemente afectadas, destacaron los dedos. Por tanto, es necesario hacer mayor hincapié en sensibilizar a los directivos y trabajadores sobre los riesgos laborales y sus consecuencias, con miras a reducir la exposición a los riesgos y posibles daños a la salud.

Palabras clave: Hospitales; Ergonomía; Salud laboral; Enfermería.

\section{Introdução}

Trabalhadores da enfermagem realizam suas atividades em diversos locais, dentre os quais as instituições hospitalares. Nesses ambientes, algumas vezes, o trabalho ocorre de forma exaustiva, pois trabalha-se com situações que exigem atenção constante, esforço físico, posições inadequadas, movimentos repetitivos e levantamento de peso, o que predispõe ao risco de adoecimento pelo trabalho (Freire \& Costa, 2016). Assim a rotina laboral torna os profissionais expostos a diversos fatores de riscos ocupacionais, como lombalgias, devido a postura corporal inadequada, por exemplo. Além disso, é comum que os profissionais evidenciem cansaço, desânimo, dores no corpo e estresse (Farias \& Oliveira, 2012; Balthazar et al., 2017).

Além disso, o trabalho dos profissionais de enfermagem, por vezes, é estressante devido à forte carga psicológica e emocional decorrente da relação profissional de enfermagem com os pacientes, as inúmeras exigências físicas, turnos prolongados, reduzido número de trabalhadores, condições inadequadas de trabalho, baixo reconhecimento e/ou valorização, das responsabilidades com a vida humana, do contato frequente com a dor, o sofrimento e a morte (Frota et al.,2013; Borges et al., 2014; Silva, Valente \& Camacho, 2020).

Embora historicamente a classe profissional de saúde não tenha sido compreendida como de alto risco para incidentes e patologias profissionais, pesquisas afirma que os colaboradores da área da saúde estão expostos com maior frequência a riscos ocupacionais comparado a outras profissões (Cavalcante et al., 2006; Balthazar et al., 2017). Santana et al. (2016) salienta as responsabilidades dos profissionais técnicos de enfermagem que, nas instituições de saúde pública brasileiras, se encontram sobrecarregados pela grande demanda de usuários nos serviços de saúde, sendo em maior proporção nas unidades de terapia intensiva, centros cirúrgicos e serviços de pronto atendimento, uma vez que, nessas unidades é exigido do profissional maior habilidade técnica, conhecimento, raciocínio rápido e atenção, visto à gravidade dos pacientes (Santana et al., 2016). 
Neste sentido, torna-se necessário repensar nos modelos de organização dos processos de trabalho, criando condições de flexibilização através da participação dos trabalhadores nas suas decisões e transformações, favorecendo a promoção da saúde e a melhoria da qualidade de vida do trabalhador. Isso contribuirá para a diminuição dos acidentes de trabalho e, consequentemente, refletirá na minimização do adoecimento profissional (Cavalcante et al., 2006). Considera-se acidente de trabalho, segundo a lei 8.213/91, Brasil artigo 20, inciso I e II:

" Inciso I: 'doença profissional, assim entendida a produzida ou desencadeada pelo exercício do trabalho peculiar a determinada atividade e constante da respectiva relação elaborada pelo Ministério do Trabalho e da Previdência Social’. Inciso II: 'doença do trabalho, assim entendida a adquirida ou desencadeada em função de condições especiais em que o trabalho é realizado e com ele se relacione diretamente, constante da relação mencionada no inciso I' (Brasil, 1991).

Nesse contexto, destaca-se a ergonomia, conhecida como uma ciência aplicada que possui como um dos seus objetivos, prevenir e diminuir acidentes e doenças do trabalho. Assim, pode ser definida como o estudo da adaptação do trabalho ao homem ou, como a utilização de conhecimentos científicos relativos aos seres humanos, sendo necessário para a produção de ferramentas, máquinas e demais equipamentos que possam ser utilizados com o máximo de conforto, segurança e eficácia (Iida \& Buarque, 2016). Nesse sentido, a ergonomia tem um olhar voltado para a humanização do trabalho, a satisfação e um melhor desempenho entre os trabalhadores (Francisco \& Medeiros, 2016).

Vale salientar que a dor musculoesquelética foi por tempos, relacionada, especialmente, a fatores de risco, como levantamento de peso, adoção de posturas inapropriadas e ao trabalho repetitivo (Magnago et al., 2008). Contudo, estressores mentais tem sido, recentemente, acrescentados como fatores de risco. Portanto, considera-se importante avaliar os fatores psicossociais do trabalho no desenvolvimento dos Distúrbios Osteomusculares Relacionados ao Trabalho (DORT) (Magnago et al., 2008). Nesse contexto, salienta-se que existem inúmeras patologias musculoesqueléticas, que são de difícil diagnóstico devido aos aspectos etiológicos que envolvem essas doenças, os quais podem ser físicos, individuais, organizacionais, socioculturais e psicossociais e, capazes de afetar profissionais em início de carreira ou aqueles com anos de serviço, nas diferentes áreas de atuação profissional (Neves, 2006; Magnago et al., 2008). Diante dessa contextualização, essa investigação se justifica, tendo em vista a necessidade de maiores discussões sobre a ergonomia entre os profissionais da saúde, principalmente a equipe de enfermagem, por permanecem maior parte do tempo no cuidado as pessoas em diferentes condições de saúde-doença.

Frente ao exposto, questiona-se: quais são as causas e ameaças mais frequentes aos profissionais de enfermagem atuantes no setor hospitalar, no que diz respeito ao risco de saúde ocupacional? Na tentativa de responder o questionamento, objetivou-se identificar as causas e ameaças mais frequentes aos profissionais de enfermagem do setor hospitalar, no que diz respeito ao risco de saúde ocupacional.

\section{Metodologia}

Trata-se de uma Revisão Narrativa da Literatura (RNL), que se constituem como publicações amplas apropriadas para descrever e discutir o desenvolvimento ou o "estado da arte" de um determinado assunto, sob o ponto de vista teórico ou contextual. Constituem, basicamente, de análise da literatura publicada em sites, livros, artigos de revistas impressas e ou eletrônicas, vídeos, manuais ministeriais, políticas públicas, anais de eventos e tudo que possa contribuir para o primeiro contato com o objeto de estudo. A RNL não necessita indicar as fontes utilizadas, a metodologia para a busca das referências e nem os critérios utilizados na avaliação e seleção dos trabalhos consultados (Brum et al., 2015). Contudo, optou-se por descrever as informações pertinentes.

Para esse estudo, inicialmente, no mês de junho do ano de 2019, realizou-se uma busca no Observatório de segurança e saúde do trabalho, um banco de dados, de livre acesso, onde é possível fazer pesquisas específicas relacionadas a temática da 
presente pesquisa. Nessa busca encontrou-se dados referentes a documentos do Ministério da Economia, Secretaria e Inspeção do trabalho (Brasil, 2003-2018) bem como do Ministério da Saúde (Brasil, 2018).

A Análise do material do Ministério do Trabalho, possibilitou apresentar os dados relativos a: 1) Distribuição Geográfica das Comunicações de Acidentes de Trabalho (CAT); 2) Estimativa de subnotificação de acidentes de trabalho; 3) Setores econômicos com maiores números de notificações; 4) Ocupações dentro do setor hospitalar; 5) Despesas previdenciárias-auxílio doença por acidentes de trabalho.

Já, a partir da análise do Material do Ministério da Saúde (Brasil, 2018), foi possível a obtenção dos seguintes dados: 1) Lesões mais frequentes; 2) Lesões mais frequentes, considerando o mobiliário, impacto contra a pessoa/objeto e esforço físico; 3) Partes do corpo mais frequentemente atingidas; 4) Partes do corpo mais frequentemente atingidas pelo esforço físico; e, 5) Afastamento conforme a classificação Internacional de doenças. Os dados extraídos nessa busca, foram expostos em gráficos para facilitar a sua visualização e posterior discussão.

Após, realizou-se, no mês de julho de 2019, uma busca na literatura, utilizando-se os descritores: Hospitais and Ergonomia and Saúde do trabalhador and Enfermagem, onde selecionou-se 10 estudos, relacionados as questões ergonômicas em profissionais de enfermagem (quadro 1), os quais foram lidos e utilizados para discutir os dados obtidos no observatório. A literatura citada nos artigos selecionados, quando pertinente, também foi lida e utilizadas na discussão dos dados dessa pesquisa.

\section{Resultados e Discussão}

Inicialmente, apresenta-se no Quadro 1, os 10 artigos evidenciados na busca da literatura realizada para a discussão dos dados. Posteriormente, apresentam-se os dados encontrados na busca no Observatório de segurança e saúde do trabalho.

Quadro 1 - Relação dos materiais selecionados para a discussão.

\begin{tabular}{|l|c|}
\hline \multicolumn{1}{|c|}{ Título } & Ano \\
\hline $\begin{array}{l}\text { Aspectos ergonômicos na transferência de pacientes: um estudo realizado com trabalhadores de uma central de } \\
\text { transportes de um hospital universitário }\end{array}$ & 2001 \\
\hline Prevalência de sintomas musculoesqueléticos em trabalhadoras de enfermagem & 2003 \\
\hline Riscos ocupacionais do trabalho em enfermagem: uma análise contextual & 2006 \\
\hline $\begin{array}{l}\text { LER: trabalho, exclusão, dor, sofrimento e relação de gênero. Um estudo com trabalhadoras atendidas num serviço } \\
\text { público de saúde. }\end{array}$ & 2006 \\
\hline Estresse, aspectos psicossociais do trabalho e distúrbios musculoesqueléticos em trabalhadores de enfermagem & 2008 \\
\hline Cargas de trabalho, processos de desgaste e absenteísmo-doença em enfermagem. & 2013 \\
\hline Cargas de trabalho e desgastes dos trabalhadores de enfermagem de um hospital-escola & 2015 \\
\hline Indicadores de saúde dos trabalhadores da área hospitalar & 2016 \\
\hline Ergonomia: projeto e produção & 2016 \\
\hline Acidentes de trabalho no brasil: revisão bibliográfica no âmbito hospitalar & 2017 \\
\hline
\end{tabular}

Fonte: Dados da investigação (2019).

Optou-se, por realizar, primeiramente, uma análise dos acidentes de trabalho no Brasil de uma maneira ampla, considerando todas as atividades em que se tem uma relação de emprego. Em seguida traçou-se uma comparação com o setor 
de atendimento hospitalar e seus trabalhadores. Por fim, comparou-se os profissionais de enfermagem com outros trabalhadores do setor de atendimento hospitalar.

Conforme dados do Observatório de Segurança e Saúde no Trabalho-Smartlab, em 2017 foram notificados e registrados no Brasil para a população com vínculo de emprego regular, 549.405 mil acidentes de trabalho. Já, em 2018, houve um aumento de 13\%, uma vez que se registrou um total de 623,8 mil. Salienta-se, ainda, que no ano de 2018 houve um aumento em relação aos três anos anteriores, dado que demonstra a necessidade de continuar investindo em práticas de segurança e de boas condutas durante o exercício da profissão (Brasil, 2018).

A estimativa de subnotificação para acidentes de trabalho que resultaram em afastamento previdenciário, também foi observado, pois muitos registros de acidentes não possuem correspondente emissão anterior na forma da lei 8.212/91, sendo gerados no momento da concessão do benefício (Brasil, 1991). Como não existe uma métrica para os casos em que não há afastamento do trabalho, o registro de acidentes no momento da concessão do benefício, acaba sendo a melhor forma de estimar subnotificações. Assim, em 2018, constatou-se que aproximadamente $24,7 \%$ do total de acidentes não foram registrados de forma legal, com uma estimativa de 154,2 mil acidentes sem a emissão da CAT (Brasil, 2018).

Nesse sentido, entende-se que a subnotificação de registros prejudica a formulação e implementação de políticas públicas de saúde, pois as mesmas poderiam ser pensadas a partir dos dados, possibilitando estratégias de promoção e prevenção dos acidentes de trabalho e doenças ocupacionais (Rinaldi, 2019). Nesse sentido, Batista et al. (2017) enfatiza que na maioria das ocorrências de acidentes de trabalho, os envolvidos são profissionais da saúde, principalmente enfermeiros, fato que decorre da exposição desses profissionais a diversos riscos que podem levar a um incidente, doença ou disfunção no trabalho. Sobre as lesões mais frequentes nas notificações de acidentes de trabalho em 2018 no Brasil, no setor hospitalar, observou-se o predomínio de lesões relacionadas: corte, laceração e ferida contusa, conforme pode ser observado no Gráfico 1.

Gráfico 1 - Lesões mais frequentes

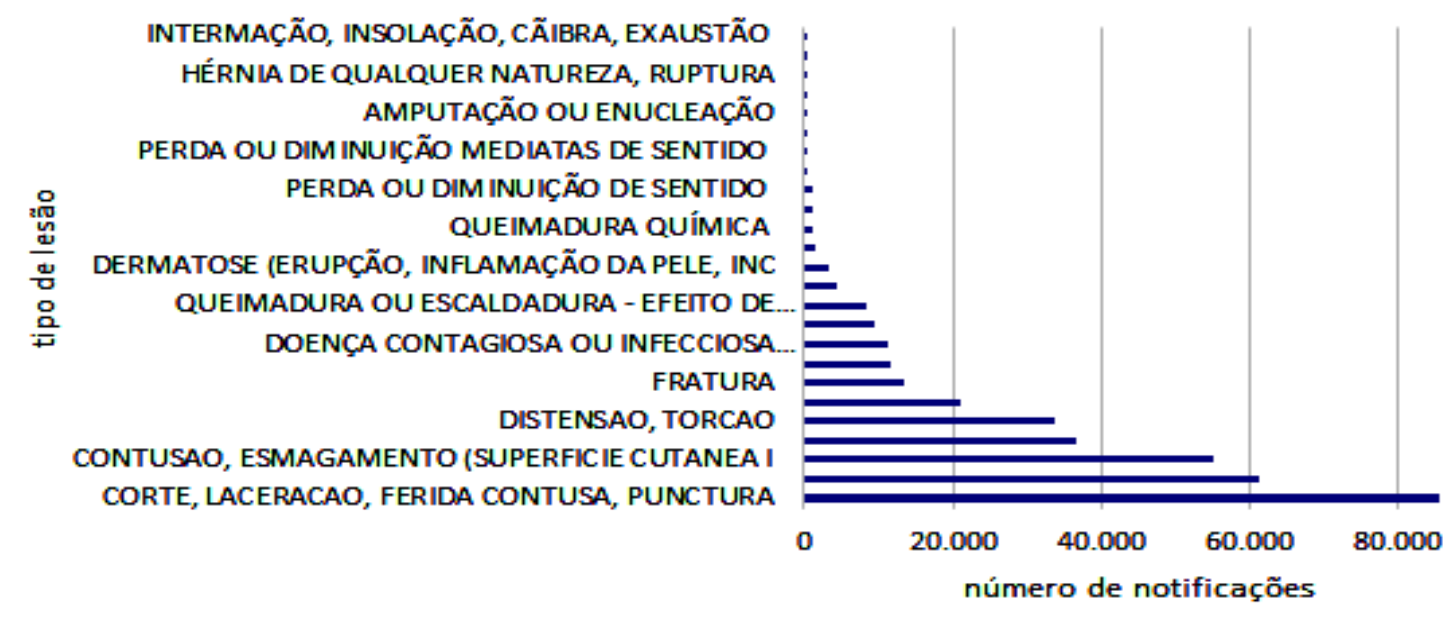

Fonte: Adaptado do Ministério da saúde (2018).

Conforme observa-se no Gráfico 1, destacaram-se as lesões decorrentes de corte, laceração e ferida contusa, bem como punctura. Pesquisa desenvolvida em Curitiba -PR, com 1.360 profissionais de enfermagem, identificou dado semelhante, uma vez que demonstrou que $61 \%$ dos eventos vivenciados pelos profissionais foram decorrentes da exposição às cargas mecânicas. Destes, associados à carga biológica, 43,5\% foram por meio dos acidentes com perfurocortantes (Karino et al., 2015). 
Com relação a parte do corpo mais atingida segundo as notificações de acidentes de trabalho no último ano, apurado no Brasil, constatou-se um predomínio nas lesões de dedos, seguidas das lesões nas mãos, nos olhos, pés e nos joelhos, conforme pode ser observado no Gráfico 2.

Gráfico 2 - Partes do corpo mais frequentemente atingidas.

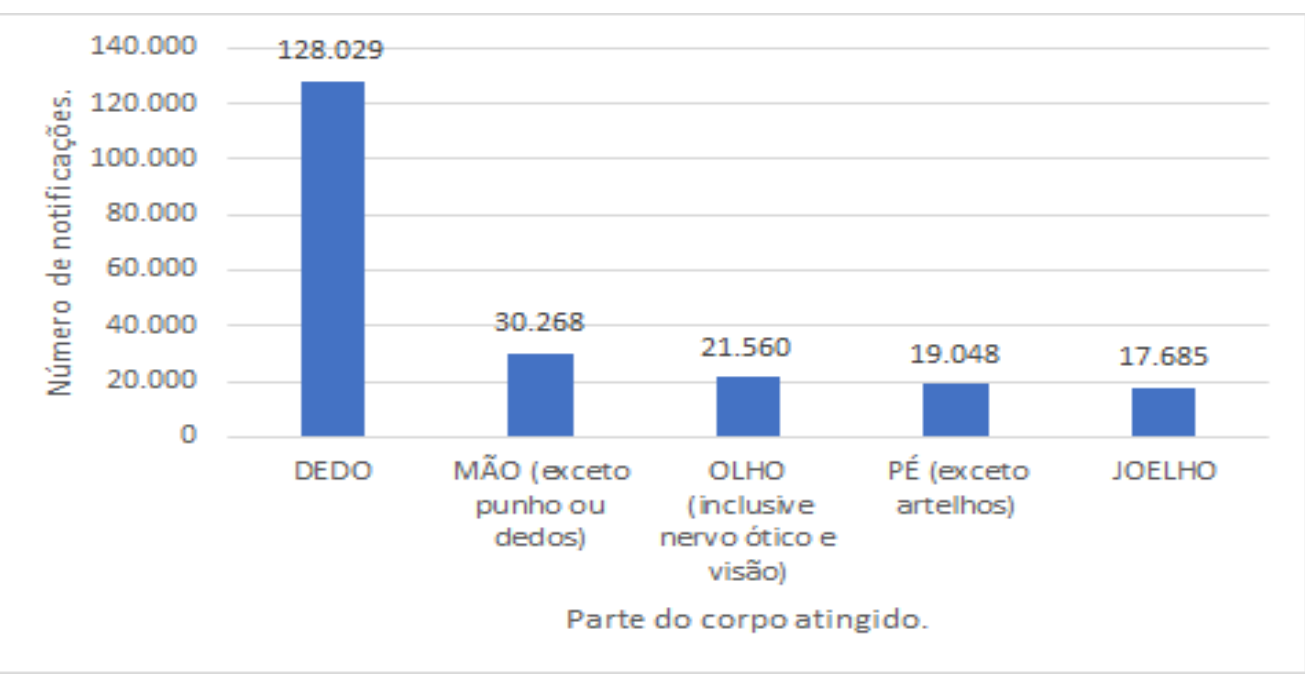

Fonte: Adaptado do Ministério da Saúde (2018).

Esse dado vem ao encontro de uma pesquisa desenvolvida na região nordeste do Brasil, com objetivo de identificar as características sociodemográficas e o perfil dos acidentes de trabalho entre os 45 profissionais de enfermagem de uma Unidade de Terapia Intensiva. Na pesquisa em questão, constatou-se que a região corporal com maior ocorrência de acidentes foram os dedos (35,6\%), seguido das mãos (13,4\%), olhos (6,6\%) e por último os pés (4,4\%) (Mendonça et al., 2015).

Em destaque, à ocupação mais frequentemente citada em notificações de acidentes de trabalho no último ano apurado no Brasil, considerado o universo de trabalhadores com vínculo de emprego, tem-se o Gráfico 3, no qual comparam-se, para o período de 2018, os percentuais de participação das diferentes ocupações nas notificações de acidentes, no setor de atendimento hospitalar. 


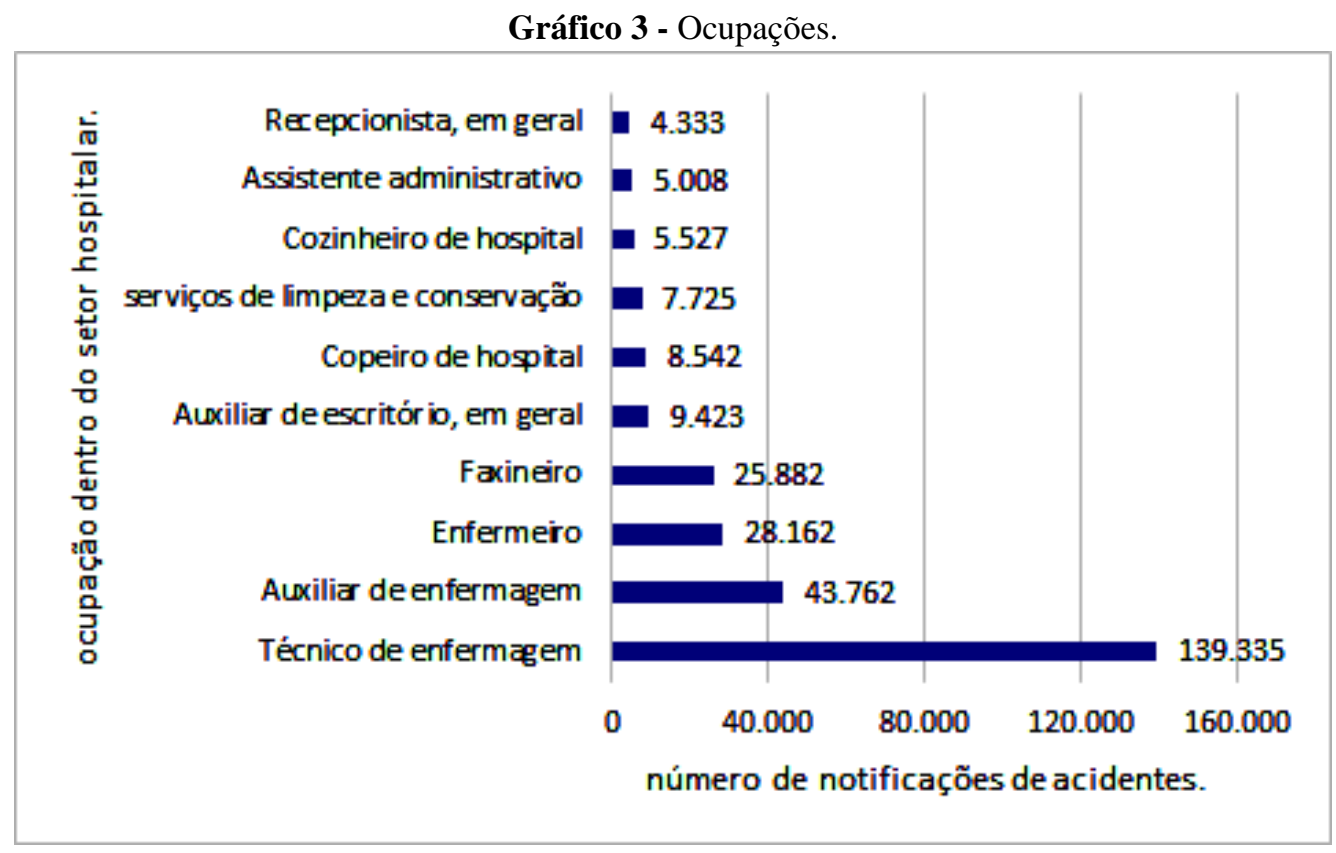

Fonte: Adaptado ministério da economia, secretaria de inspeção do trabalho (2003-2018).

Observa-se no Gráfico 3, que somando o percentual dos profissionais de enfermagem (enfermeiro, técnico de enfermagem e auxiliar de enfermagem) tem-se aproximadamente $60 \%$ dos casos de acidentes dentro da unidade hospitalar. Entende-se que o número expressivo de acidentes de trabalho na enfermagem é possibilitado por alguns fatores, como: sobrecarga de tarefas, jornada de trabalho, cansaço, estresse, condições insalubres e o desconhecimento e/ou desinteresse dos profissionais sobre esta temática (Mendonça et al., 2015).

Somado a isso, tem-se o fato dos técnicos representam a categoria mais numerosa entre os profissionais de enfermagem e suas atividades são em grande parte relacionadas à assistência direta ao paciente. Esse fato pode justificar o maior número de mais acidentes nesse grupo de profissionais (Gallas \& Fontana, 2010). Além disso, o risco ergonômico parece subestimado desde a formação da enfermagem ou não compreendido pelo profissional, visto a preocupação maior na prevenção do risco biológico, por exemplo (Dias et al., 2020).

Em relação aos motivos dos afastamentos do ambiente laboral dos profissionais de enfermagem do setor hospitalar, entre os anos de 2012 a 2018, no Brasil, apresenta-se o Gráfico 4. 
Gráfico 4 - Afastamentos conforme a Classificação Internacional de Doenças.

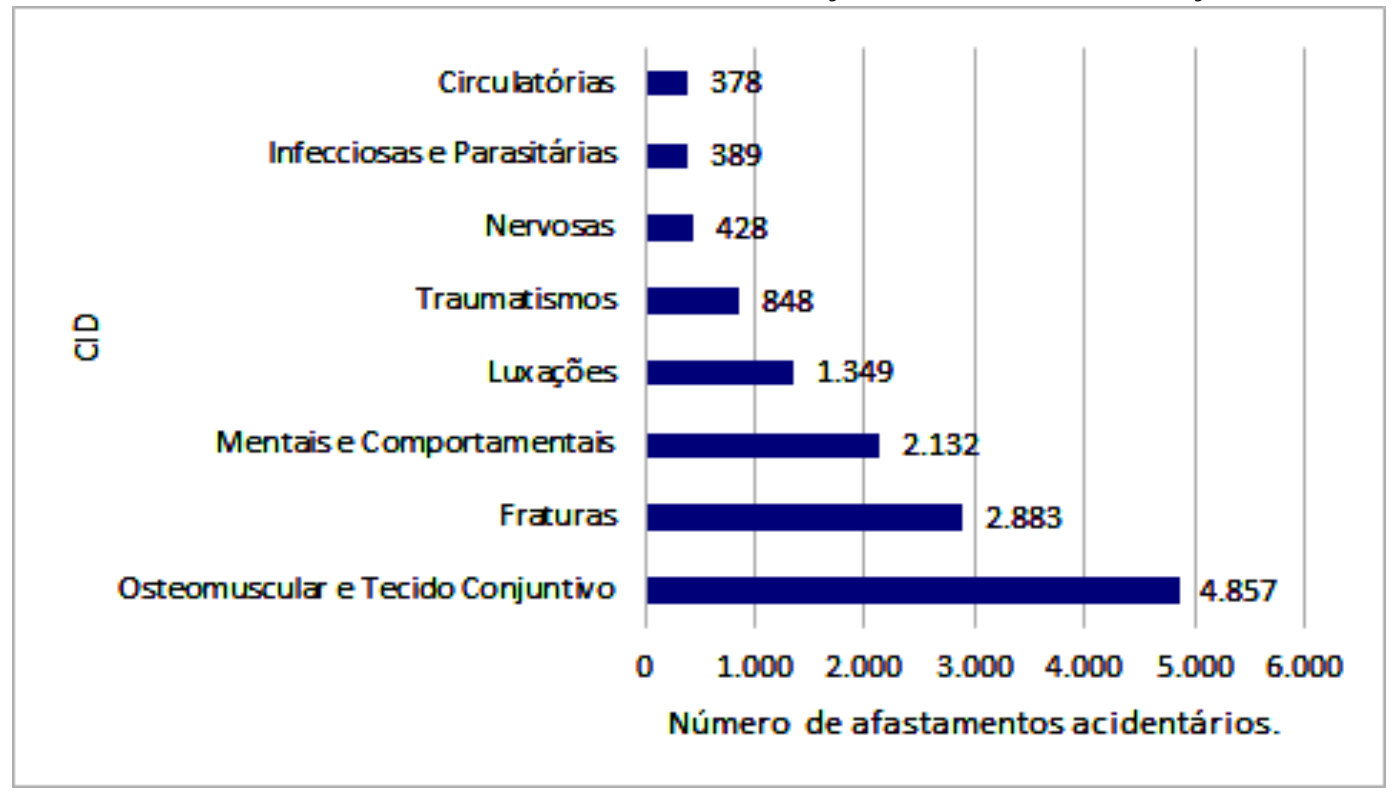

Fonte: Adaptado do Ministério da saúde (2018).

Como observa-se no Gráfico 4, houve destaque para as alterações ósseas, osteomusculares e de tecido conjuntivo. Dado semelhante foi evidenciado em outras pesquisas. Pesquisa desenvolvida com 105 técnicos e auxiliares de enfermagem como objetivo de avaliar sintomas musculoesqueléticos desses trabalhadores. Na referida pesquisa, evidenciou-se que, independentemente da região afetada, $93 \%$ das participantes referiram algum tipo de sintomas osteomusculares nos últimos 12 meses, e $62 \%$ nos últimos sete dias. Nos últimos 12 meses, salientou-se alterações nas seguintes regiões anatômicas: lombar $(59 \%)$, ombros $(40 \%)$, joelhos $(33,3 \%)$ e região cervical $(28,6 \%)$. Em relação aos últimos 7 dias, destacou-se a dor na região lombar (31,4\%), seguida pelos ombros (16,2\%) (Gurgueira; Alexandre; Filho, 2003).

Corroborando com esses dados, tem-se os de uma pesquisa que objetivou analisar as cargas de trabalho, processos de desgaste e absenteísmo por doença entre trabalhadores de enfermagem de um hospital universitário, da Região Centro-Oeste do Brasil. Os dados da referida pesquisa demonstraram que as doenças do sistema osteo conjuntivo e tecido muscular constituem o principal problema de saúde, com $21 \%$ das notificações, seguidos dos transtornos mentais e comportamentais (15,3\%) dos profissionais (Mininel et. al., 2013).

Além disso, observa-se, que transtornos mentais como a depressão podem se desencadear nos profissionais de saúde. Isto ocorre devido a diversos fatores, tais como, excessiva jornada de trabalho, pouco reconhecimento, estresse e tensão no trabalho, assim influenciando na saúde e na qualidade da assistência, além de colaborar, ano após ano, para um aumento no prejuízo da previdência social do Brasil (Alves et al., 2019). A esse respeito, a pesquisa que objetivou relacionar as cargas de trabalho e o processo de desgaste ao adoecimento dos trabalhadores de enfermagem de um hospital escola, demonstrou que dos $2,1 \%$ dos eventos relacionados às cargas psíquicas, $17,4 \%$ foram devido às depressões e sofrimentos pelos excessos de atividades no trabalho (Karino et al., 2015).

Diante ao exposto, ficou evidente com a análise dos dados a constante exposição dos profissionais da saúde aos riscos ergonômicos. Isto geralmente é consequência da postura inadequada para realização das atividades diárias e pelo excesso de trabalho. Concomitante a esses riscos, surgem os riscos psicológicos que são desencadeados pelas condições insalubres que os profissionais no ambiente de trabalho, baixa remuneração, reduzido número de colaboradores, entre outros. 
Assim, destaca-se que um dos desafios da enfermagem, a reorientação da sua prática profissional dentro da perspectiva de transformação do seu processo de trabalho, com vistas à diminuição do impacto da sua divisão social e da sua organização que interferem no desgaste físico e psíquico do trabalhador (Cavalcante et al., 2006).

\section{Conclusão}

A realização desse estudo foi satisfatória, pois permitiu identificar as causas e ameaças mais frequentes aos profissionais de enfermagem do setor hospitalar, no que diz respeito ao risco de saúde ocupacional.

Identificou-se que somados os percentuais de lesões sofridas por profissionais de enfermagem (enfermeiro, técnico de enfermagem e auxiliar de enfermagem), tem-se aproximadamente $60 \%$ dos casos de acidentes ergonômicos dentro da unidade hospitalar.

Quanto as causas mais frequentes de afastamentos dos profissionais do ambiente hospitalar, a maior incidência foi em decorrência de lesões osteomusculares e de tecido conjuntivo, seguida de fraturas, doenças mentais e comportamentais, bem como luxações. Quanto as partes do corpo mais frequentemente atingidas, destacou-se os dedos. Quando analisado as partes do corpo mais frequentemente atingidas por esforço físico, evidenciou-se maior incidência nas lesões em ombros, dorso, punho e membros superiores.

Foi possível identificar que dentre as ameaças ergonômicas a que estão expostos os profissionais, destacam-se as atividades e cuidados hospitalares contínuos aos pacientes, a sobrecarga em consequência da alta demanda de usuários que buscam os serviços de saúde, o estresse gerado pelo ambiente de trabalho, que influência tanto na saúde física quanto psíquica dos profissionais, assim possibilitando o descontentamento, a grande rotatividade de profissionais no serviço e o absenteísmo, o que gera redução na qualidade do atendimento.

Assim faz-se necessário maior ênfase na sensibilização desses trabalhadores a respeito dos riscos ocupacionais e suas consequências, como também, sobre as normas existentes em legislação, pois entende-se que os profissionais precisam se perceber como parte importante na reversão desse quadro e responsáveis pelo processo de qualidade no trabalho, reduzindo a exposição a riscos e possíveis danos à saúde.

Além disso, os gestores das instituições precisam estar atentos as questões que proporcionam maior risco a saúde dos trabalhadores para implementar medidas com vista a reduzi-los. Uma vez que, é notória a necessidade que sejam realizados investimentos nas áreas de gestão do conhecimento e das boas práticas hospitalares, assim estimulando a realização de atividades pelos profissionais de enfermagem, estes que apresentam maiores índices de acidentes no trabalho, como também, médicos e demais colaboradores.

Espera-se que este estudo, venha a contribuir com subsídios para quem busque informações sobre o assunto. Considera-se que o mesmo pode ser utilizado em diferentes tipos de profissões e não somente na área da enfermagem, pois como já citado, todos os profissionais, em maior ou menor gravidade, estão expostos a riscos ergonômicos.

\section{Referências}

Alves, A., Carvalho, V. C. S., Santos, M. S., Oliveira, J. A. A., Gomes, M. F. P., Reticena, K. O., Bravo, D. S. \& Oliveira, J. (2019). Depressão entre profissionais de enfermagem no contexto hospitalar: Uma Revisão De Literatura. Brazilian Journal of Surgery and Clinical Research, 27(3),141-146.

Balthazar, M. A. P., Andrade, M., Souza, D. F., Cavagna, V. M. \& Valente, G. S. C. (2017). Gestão dos riscos ocupacionais nos serviços hospitalares: uma análise reflexiva. Rev Enferm UFPE, 11(9), 3482-91. doi: 10.5205/reuol.11088-99027-5-ED.1109201720.

Batista, E. S., Ferreira, D. N. S., Santos, J. O. \& Góes, A. O. S. (2017). Acidentes de trabalho no brasil: revisão bibliográfica no âmbito hospitalar. C@leaCadernos de aulas do LEA. (6), 81-92.

Borges, T. P., Kurebayashi, L. F. S. \& Silva, M. J. P. (2014). Lombalgia ocupacional em trabalhadores de enfermagem: massagem versus dor. Revista da Escola de Enfermagem da USP, 48(4), 669-675. 
Brasil. (1991a). Lei n. 8.212 de 24 de julho de 1991. Lei orgânica da seguridade social. Conceituação e princípios constitucionais. Presidência da República.

Brasil. (1991b) Lei n. 8.213 de 24 de julho de 1991. Da finalidade e dos princípios básicos da previdência social. Presidência da República.

Brasil. (2005). Ministério do Trabalho e Emprego Portaria n. 485, de 11 de novembro de 2005. Aprova a Norma Regulamentadora n. 32 - Segurança e saúde no trabalho em estabelecimentos de saúde. Diário Oficial [da] República Federativa do Brasil, Brasília, DF.

Brasil. (2018). Ministério da Economia, secretaria de inspeção do trabalho. Anuário estatístico de acidentes do trabalho.

Brasil. (2018). Ministério da Saúde. Sistema de Informação de Agravos de Notificação - Sinan.

Brasil. (2018). Observatório digital de Saúde e Segurança do trabalho - Smartlab. Disponível em: < https://observatoriosst.mpt.mp.br/ >. Acesso em 15 de junho de 2019.

Brum, C. N., Zuge, S. L. S., Rangel, R. F., Freitas, H. M. B. \& Pieszak, G. M. (2015). Revisão narrativa: aspectos conceituais e metodológicos na construção do conhecimento da enfermagem. In: Costenaro R, Lacerda MR. Metodologia da pesquisa para a enfermagem e saúde. Porto Alegre: Moriá. p.124-142.

Cavalcante, C. A. A., Enders, B. C., Menezes, R. M. P. \& Medeiros, S. M. (2006). Riscos Ocupacionais do Trabalho em Enfermagem: Uma Análise Contextual. Ciência, Cuidado e Saúde, 5(1), 88-97.

Dias, E. G., Souza, S. P. D., Gomes, J. P., Caldeira, M. B. \& Teixeira, J. A. L. (2020). Riscos ergonômicos do ambiente de trabalho do enfermeiro na atenção básica e no pronto atendimento. J. nurs. Health, 10(2), e20102004.

Farias, G. S. \& Oliveira, C. S. (2012). Riscos ocupacionais relacionados aos profissionais de enfermagem na UTI: uma revisão. Brazilian Journal of Health, $3(1), 1-12$.

Francisco, W. G. \& Medeiros, A. P. (2016). Análise ergonômica: estudo de caso do depósito de uma empresa de materiais para construção da Grande Florianópolis. DA Pesquisa, 11(15), 271-90.

Freire, M. N. \& Costa, R. E. (2016). Qualidade de vida dos profissionais de enfermagem no ambiente de trabalho. Revista enfermagem contemporânea, 5(1), $151-8$.

Frota, N. M., Barros, L. M., Caldini, L. N., Araújo, T. M. \& Caetano, J. Á. (2013). Saúde ocupacional dos profissionais de enfermagem em unidade de terapia intensiva. Enfermagem em Foco, 4(2), 115-118.

Gurgueira, G.P., Alexandre, N.M.C., Filho, H.R.C. (2003). Prevalência de sintomas músculo-esqueléticos em trabalhadoras de enfermagem. Rev Latino-am Enfermagem, 11(5), 608-13.

Karino, M.E., Felli, V.E.A., Sarquis, L.M.M., Santana, L.L., Silva, S.R.,Teixeira, R.C. (2015). Cargas de trabalho e desgastes dos trabalhadores de Enfermagem de um hospital-escola. Cienc Cuid Saude, 14(2), 1011-1018.

Lida, I. \& Buarque, L. (2016). Ergonomia: Projeto e Produção. 3. ed. São Paulo: Blucher.

Magnago T. S. B. S., Lisboa M. T. L. \& Griep R. H. (2008). Estresse, aspectos psicossociais do trabalho e distúrbios musculoesqueléticos em trabalhadores de enfermagem. Revista Enfermagem UERJ, 17(1), 118-123.

Mendonça, A. E. O., Oliveira, A. V. T., Neto, V. L. S. \& Silva, R. A. R. (2015). Perfil de acidentes de trabalho envolvendo profissionais de enfermagem no ambiente da Terapia Intensiva. Enfermería Global, (39), 202-210.

Mininel, V. A., Felli, V. E. A., Silva, E. J., Torri, Z., Abreu, A. P. \& Branco, M. T. A. (2013). Cargas de trabalho, processos de desgaste e absenteísmo-doença em enfermagem. Revista Latino Americana de Enfermagem, 21(6), 1290-1297.

Neves, I. R. (2006). LER: trabalho, exclusão, dor, sofrimento e relação de gênero. Um estudo com trabalhadoras atendidas num serviço público de saúde. Caderno de Saúde Pública, 22(6), 1257-1265.

Rinaldi, A. (2019). Subnotificação dificulta implementação de políticas públicas. http://www.fundacentro.gov.br/noticias/detalhe-danoticia/2019/8/subnotificacao-dificulta-implementacao-de-politicas-publicas.

Rossi, C. G., Rocha, R. M. \& Alexandre, N. M. C. (2001). Aspectos ergonômicos na transferência de pacientes: um estudo realizado com trabalhadores de uma central de transportes de um hospital universitário. Revista da Escola de Enfermagem da USP., 35(3), $242-248$.

Santana, L. L., Sarquis, L. M. M., Miranda, F. M. A., Kalinke, L. P., Felli, V. E. A. \& Mininel, V. A. (2016). Indicadores de saúde dos trabalhadores da área hospitalar. Revista Brasileira de Enfermagem, 69(1), 30-39.

Silva, R. P. S., Camacho, A. C. L. \& Valente, G. S. C. (2020). Risk management in the scope of nursing professionals in the hospital setting. Rev Bras Enferm, 73(6), e20190303. 